\title{
Changes in Electroencephalographic Results and Heart Rate Variability after Exposure to Green Landscape Photographs Correlated with Color Temperature and Illumination Level
}

\author{
Min Jung Lee ${ }^{1}$ and Wook $\mathrm{Oh}^{2,3^{*}}$ \\ ${ }^{1}$ Research Professor, Department of Horticulture \& Life Science and Rural Development Institute, Yeungnam University, Gyeongsan \\ 38541, Republic of Korea \\ 2Professor, Department of Horticulture \& Life Science, Yeungnam University, Gyeongsan 38541, Republic of Korea \\ ${ }^{3}$ Directer, Rural Development Institute, Yeungnam University, Gyeongsan 38541, Republic of Korea
}

\section{ABSTRACT}

Background and objective: Various images from visual display terminals (VDTs) as well as living lighting are important parts of our daily life; thus, properly controlling the lighting environment - that is, illuminance, color temperature and good images from VDTs - can have a substantial effect on improving the mental health and work efficiency in everyday life. We examined electroencephalography (EEG) and heart rate variability (HRV) responses to various lighting conditions in 25 university students as they viewed images of a green landscape or traffic congestion.

Methods: EEG was performed in darkness and when the room was illuminated with 10 different light-emitting diode (LED) color temperatures, while the EEG and HRV responses to green landscape or traffic congestion image stimuli were measured in darkness and during room illumination with three different LED color temperatures.

Results: We found a significant difference between darkness and high LED illumination (400 Ix) at 7 (CZ, F4, FZ, O1, O2, $\mathrm{OZ}$, and T6) of 30 channels, while the alpha wave activity increased during darkness. In the second experiment, the green landscape image stimuli in the $30 \mathrm{~lx}-2600 \mathrm{~K}$ lighting condition elicited theta wave activity on the EEG, whereas the traffic congestion image stimuli under high LED illumination elicited high beta and gamma wave activities. Moreover, the subjects exhibited better stress coping ability and heart rate stability in response to green landscape image stimuli under illuminated conditions, according to their HRV.

Conclusion: These results suggest that lower color temperatures and illumination levels alleviate tension, and that viewing green landscape image stimuli at low illumination, or in darkness, is effective for reducing stress. Conversely, high illumination levels and color temperatures are likely to increase tension and stress in response to traffic congestion image stimuli.

Keywords: greenery image, light-emitting diode, lighting environment, urban landscape, visual display terminals

\section{Introduction}

Light emitting diodes (LEDs) have a longer life and higher energy efficiency and are more environmentally friendly than other lighting devices currently used, such as fluorescent lamps, incandescent lamps, and halogen bulbs (KEIT, 2013). Consequently, the development and use of LEDs as an alternative light source has been accelerated in Korea and overseas. In our society today, individuals spend long periods of time indoors, and research has begun to address the effects of environmental factors in indoor spaces on psychological and physiological

Abbreviations: Electrocardiograph (ECG), Electrodermal activity (EDA), Electroencephalographic (EEG), Heart rate (HR), Heart rate variability (HRV), Light-emitting diodes (LEDs), Low frequency to high frequency ratio (LF/HF), Root mean square of the standard deviation (RMSSD), Standard deviation of the N-N interval (SDDN)

This study was supported by a 2014 Yeungnam University Research Grant.

Received: November 6, 2021, Revised: November 17, 2021, Accepted: November 24, 2021

First author: Min Jung Lee, pepern@naver.com, (D) https://orcid.org/0000-0002-5262-0389

*Corresponding author: Wook Oh, wookoh@ynu.ac.kr, (D) https://orcid.org/0000-0002-2552-5511 
well-being. Lighting is one such factor that can have substantial effects on psychological and physiological status.

The color temperature represents the color of the light source and depends on the heating of a perfect radiator (black body) that emits colored light at high temperatures. The color of light changes with temperature. Low color temperatures produce high-intensity red-colored light, high color temperatures produce white-colored light, and extremely high temperatures of $15,000 \mathrm{~K}$ or above produce blue-colored light (KCIE, 2009). Manav (2007) studied participants' subjective responses to changes in the illumination level and color temperature and found that higher illumination levels were preferred, and that a color temperature of 4,000 $\mathrm{K}$ was associated with higher preference and satisfaction compared with a color temperature of 2,700 K for impressions of 'comfort and spaciousness.'

Multiple biosignals are used to assess human psychological and physiological responses to external stimuli, such as electroencephalography (EEG) and electromyographic signals, electrodermal activity (EDA), and heart rate (HR). Spontaneous EEG signals, event-related potentials, and electrocardiographic signals, such as changes in color and light, have been used to study responses to visual stimuli, such as changes in color and light (Jung et al., 2010). An increasing number of studies have employed these measures to estimate the psychological responses to external stimuli, such as color, light, smell, and sound (Zion-Golumbic et al., 2008; Ergenoglu et al., 2004; Lang and Bradley, 2010; Schneider et al., 2009; Chebat and Michon, 2003; Martin, 1998). A substantial body of literature suggests that lighting attributes, such as color, brightness, and chroma, can affect physiological phenomena (Kaiser, 1984), behavioral performance (Stone, 2001), sleep timing (Higuchi et al., 2021; Sunwoo et al., 2017), and psychological responses (Suk and Irtel, 2010; Suk et al., 2012; Park et al., 2013); however, only a few studies have assessed how illumination levels and the colors of LED light sources affect individual psychological status. Psychological and physiological studies are required to determine the emotional and psychological effects of LED lighting, especially in the context of various user activities.

Because of the current coronavirus (COVID-19) pandemic, the length of time that humans spend indoors has increased significantly. Therefore, the indoor environment has an even greater influence on human emotions, and the importance of indoor lighting is subsequently increasing. As the daily use of visual display terminals (VDTs) such as TVs, PCs, and smartphones increases, the amount of time spent in nature decreases. During the COVID-19 pandemic, restrictions due to social distancing have closed public green spaces and kept people at home (Chae et al., 2021), and people have had to face a lot of stress during this forced isolation period (Lee and Yeon, 2021). The pandemic has also changed the usage patterns of green areas (Chae et al., 2021; Lee and Yeon, 2021). As a result, the awareness and importance of green spaces has increased significantly (Larcher et al., 2021; Bulgari et al., 2021). We speculated whether viewing greenery images on a screen could have an effect similar to that of viewing actual greenery, and whether this was affected by the type of indoor lighting. It was thus also necessary to determine the effect of watching green natural areas such as gardens, parks, and forests through indoor devices (visual display terminals) for a certain period of time.

In the present study, we conducted two experiments to examine human brain activity in differently illuminated environments in the presence and absence of visual stimuli (Parsons, 2012; Mulholland, 1973). First, we measured the EEG signals of 24 university students during environmental illumination at various LED color temperatures. In the second experiment, we measured the HR variability (HRV) and EEG signals in the same subjects in response to green landscape (positive) and traffic congestion (negative) visual stimuli under different LED lighting conditions. In this way, we sought to determine the optimal LED color temperature for different human activities.

\section{Research Methods}

\section{Subjects}

To recruit subjects, we announced this study on the online student bulletin board within Yeungnam University, and asked students who voluntarily expressed their intention to participate in an individual interview to fill out 
a questionnaire, while ensuring that normal conversation with these students was possible. We fully explained the research plan and activities to the students, and then conducted the research on the students who signed the consent form. We enrolled 24 university students (13 males and 11 females) with a mean age of 21 years who were free of chronic, cardiac, and lung diseases and had normal color vision with acuity corrected to 0.8 or higher. One day before the experiment, the subjects were instructed regarding the experiment's procedure and were asked not to drink or exercise in excess. They were also instructed to refrain from eating or consuming caffeine for at least $4 \mathrm{~h}$ before the experiment.

\section{Equipment}

\section{EEG measurement}

Curry 7 (Neuroscan Inc., Charlotte, NC, USA), consisting of a Synamps2 system and a 32-channel Quick Cap, was used to measure the EGG signals. The ground connection was attached at Fpz of the forehead, and M1 and M2 were attached to the mastoid behind the ears as reference electrodes. Vertical electrooculographic signals were recorded from above and below the right eye to compensate for eye blinking and horizontal electrooculographic signals from the lateral canthi of both eyes. An echocardiograph (ECG) was used to compensate for noisy signals. The electrode resistance value was $10 \mathrm{k} \Omega$, the band-pass filter value was $0.1-40.0 \mathrm{~Hz}$, and the sampling rate was $1,000 \mathrm{~Hz}$. The EEG signals were analyzed by extracting the values of relative power in Neuroguide (Applied Neuroscience Inc., Largo, FL, USA).

\section{HRV measurement}

One of the Bionomadix ECG2 series of wireless, wearable monitoring devices (MP150 System by Biopac) was used to measure HRV and EDA. The AcqKnowledge 4.4 software was used to record and store the data. The values extracted from the ECG2 were used to obtain the standard deviation of the $\mathrm{N}-\mathrm{N}$ interval (SDNN), HR, root mean square of the standard deviation (RMSSD), and low frequency to high frequency ratio using Kubios HRV 2.1 software. The SDNN is an index that represents the rate of change in HR; a higher SDNN indicates better health, while a lower score indicates the loss of stress-coping ability and impaired regulation of the autonomic nervous system. The RMSSD is the square root of the mean of the sum of the squares of the differences between adjacent $\mathrm{N}-\mathrm{N}$ intervals, obtained by calculating the mean after calculating the differences between adjacent $\mathrm{N}-\mathrm{N}$ intervals and then squaring each to obtain the square root of the total. The RMSSD accordingly shows short-term variances in $\mathrm{HR}$, and is used to evaluate the variance among pulses in the high-frequency area of HRV, which indicates parasympathetic cardiovascular regulation (i.e., high values indicate activation of the parasympathetic nervous system). Therefore, this index represents HR stability.

\section{Experimental Conditions and Procedures}

The experiments were conducted in a shielded room with white walls and three Viola 90 color-changing LED lights (Feelux) installed on the ceiling. Lighting was controlled by a PC using Magic Q 100Pro software (Chamsys). The environmental conditions produced by the colored LED lights were measured using a CL-500 Chroma/Light Meter (Konica Minolta).

The experiments examined two levels: 1) LED color temperature and 2) positive (green landscape) and negative (traffic congestion) images. The experimental setup is shown in Fig. 1.

\section{Experiment 1}

As shown in Table 1 and Fig. 2, the environmental characteristics were measured under 11 color temperature lighting conditions. A $30 \mathrm{~s}$ rest in darkness was provided to each participant before exposure to different light stimulus under random lighting conditions for $30 \mathrm{~s}$. Fig. 3A shows the experimental procedure.

\section{Experiment 2}

As shown in Table 2, environmental characteristics were measured under a few lighting conditions after presenting positive (green landscape) and negative (traffic congestion) 

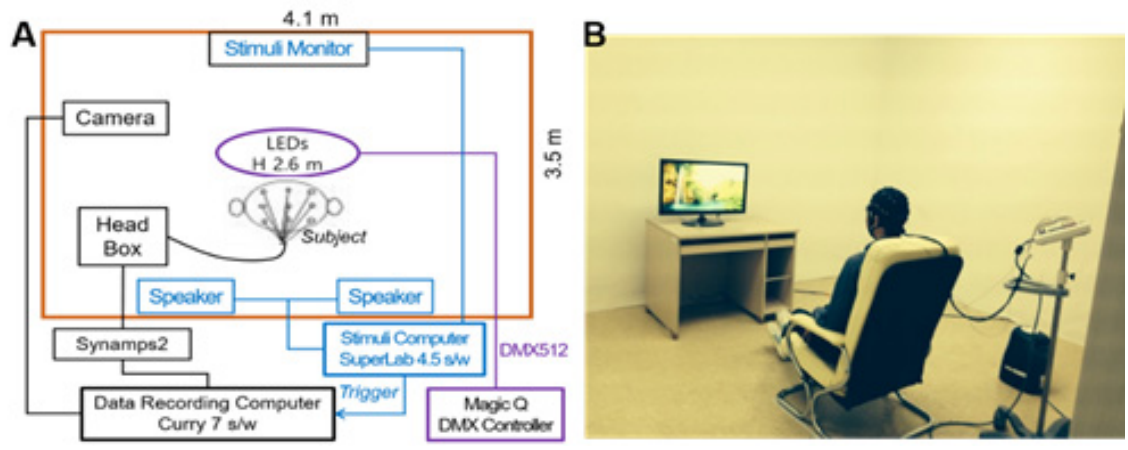

Fig. 1. Experimental set-up: Inside the shielded laboratory $(4.1 \mathrm{~m} \times 3.5 \mathrm{~m} \times 2.6 \mathrm{~m})$, with an observation camera, light-emitting diode (LED) lighting, stimulus presentation monitor, and wired EEG headbox, and an external SuperLab s/w computer connected to an internal monitor, wired EEG Data recording (Curry $7 \mathrm{~s} / \mathrm{w}$ ) computer, and shielded room lighting control (Magic Q) computer.

Table 1. Lighting conditions for Experiment 1

\begin{tabular}{|c|c|c|c|c|c|c|c|}
\hline \multirow{3}{*}{ No. } & \multirow{3}{*}{ Lighting label } & \multicolumn{2}{|c|}{$\mathrm{CIE}^{\mathrm{z}}$} & \multirow{3}{*}{$\mathrm{Ra}^{\mathrm{y}}$} & \multirow{3}{*}{$\begin{array}{c}\text { Dominant } \\
\text { wavelength }^{\mathrm{x}} \\
(\mathrm{nm})\end{array}$} & \multirow{3}{*}{$\begin{array}{c}\text { Peak wavelength }{ }^{\mathrm{w}} \\
(\mathrm{nm})\end{array}$} & \multirow{3}{*}{$\begin{array}{c}\text { Excitation } \\
\text { purity }^{\mathrm{v}} \\
(\%)\end{array}$} \\
\hline & & \multicolumn{2}{|c|}{ Chromaticity coordinates values } & & & & \\
\hline & & $\mathrm{x}$ & $\mathrm{y}$ & & & & \\
\hline 1 & Darkness & 0 & 0 & 0 & 0 & 0 & 0 \\
\hline 2 & $2600 \mathrm{~K}-30 \mathrm{~lx}$ & 0.466 & 0.414 & 85 & 584 & 616 & 0.641 \\
\hline 3 & $3400 \mathrm{~K}-30 \mathrm{~lx}$ & 0.402 & 0.381 & 89 & 582 & 610 & 0.352 \\
\hline 4 & $4700 \mathrm{~K}-30 \mathrm{~lx}$ & 0.353 & 0.355 & 88 & 577 & 450 & 0.126 \\
\hline 5 & $5500 \mathrm{~K}-30 \mathrm{~lx}$ & 0.331 & 0.334 & 87 & 546 & 450 & 0.283 \\
\hline 6 & $6000 \mathrm{~K}-30 \mathrm{~lx}$ & 0.322 & 0.339 & 86 & 499 & 450 & 0.329 \\
\hline 7 & $2600 \mathrm{~K}-400 \mathrm{~lx}$ & 0.465 & 0.412 & 85 & 584 & 615 & 0.635 \\
\hline 8 & $3200 \mathrm{~K}-400 \mathrm{~lx}$ & 0.416 & 0.388 & 87 & 583 & 611 & 0.416 \\
\hline 9 & $4400 \mathrm{~K}-400 \mathrm{~lx}$ & 0.361 & 0.358 & 88 & 579 & 448 & 0.160 \\
\hline 10 & $5400 \mathrm{~K}-400 \mathrm{~lx}$ & 0.333 & 0.342 & 86 & 553 & 448 & 0.264 \\
\hline 11 & $6000 \mathrm{~K}-400 \mathrm{~lx}$ & 0.323 & 0.336 & 85 & 495 & 449 & 0.325 \\
\hline
\end{tabular}

${ }^{\mathrm{z}} \mathrm{CIE}$, Commission Internationale de l'Eclairage.

${ }^{\mathrm{y}} \mathrm{Ra}$ : Color rendering index (CRI). CRI is the degree of color seen by the naked eye from two light sources: light source and sunlight. ${ }^{x}$ Dominant wavelength: Method of characterizing any light mixture in terms of monochromatic spectral light that evokes an identical perception of hue.

"Peak wavelength: The wavelength at which the spectrum reaches its highest intensity.

${ }^{v}$ Excitation purity: The distance from the illuminant point to the reference point, divided by that from the illuminant point to the spectrum locus (relative distance of an object's chromaticity coordinate from the white point to the exterior of the chromaticity diagram).

image stimuli. Rest and light stimulus was provided for 30 s prior to each randomized lighting condition. Fig. 3B shows the experimental procedure. Positive and negative images (Fig. 4) were obtained under the following conditions: 2,600 $\mathrm{K}-30 \mathrm{~lx}, 4,400 \mathrm{~K}-400 \mathrm{~lx}$, and $6,000 \mathrm{~K}-400 \mathrm{~lx}$.

\section{Statistical Analysis}

SPSS ver. 14.0 for Windows (SPSS Institute Inc.,
Chicago, IL, USA) was used for data analysis. One-way analysis of variance was conducted to identify significant differences in the quantitative EEG and HRV parameters among the different lighting environments. Duncan's multiple range test was used for post-hoc testing. Fast Fourier transform (FFT) relative power group mean values (\%) were used for quantitative EEG analyses. Statistical significance was set as $p<0.05$. 


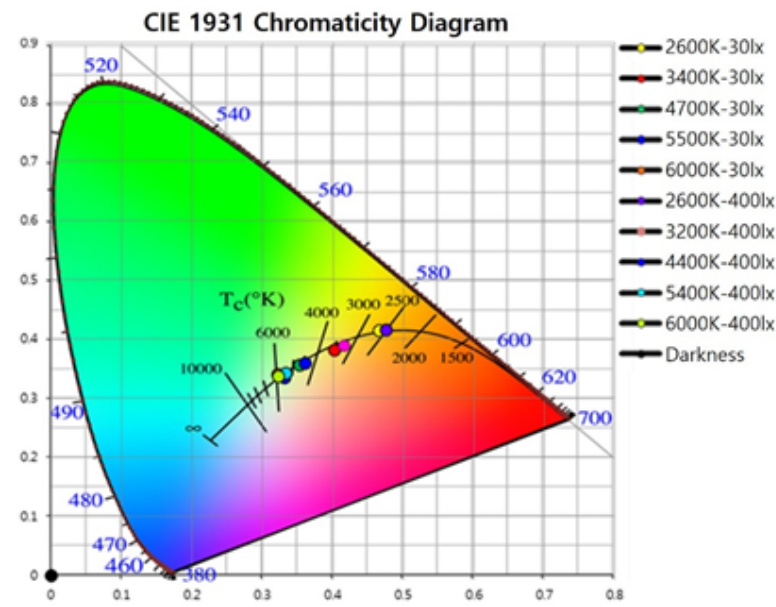

Fig. 2. CIE 1931 chromaticity diagram: This is a CIE $(x, y)$ chromaticity chart by Commission Internationale de l'Eclairage (CIE) of Planckian Locus, and 11 circles are displayed on the coordinate line including the black body locus at a temperature with a chromaticity that is not on the black body locus. Monochromatic colors are located on the perimeter, and white light is located in the center of the diagram.

A Rest Stimulus Rest Stimulus Rest Stimulus Rest Stimulus Rest Stimulus Rest Stimulus (Darkness)

\begin{tabular}{|c|c|c|c|c|c|c|c|c|c|c|c|}
\hline $30 \mathrm{~s}$ & $30 \mathrm{~s}$ & $30 \mathrm{~s}$ & $30 \mathrm{~s}$ & $30 \mathrm{~s}$ & $30 \mathrm{~s}$ & $30 \mathrm{~s}$ & $30 \mathrm{~s}$ & $30 \mathrm{~s}$ & $30 \mathrm{~s}$ & $30 \mathrm{~s}$ & $30 \mathrm{~s}$ \\
\hline Rest & Stimulus & Rest & Stimulus & Rest & Stimulus & Rest & Stimulus & Rest & Stimulus & & \\
\hline $30 \mathrm{~s}$ & $30 \mathrm{~s}$ & $30 \mathrm{~s}$ & $30 \mathrm{~s}$ & $30 \mathrm{~s}$ & $30 \mathrm{~s}$ & $30 \mathrm{~s}$ & $30 \mathrm{~s}$ & $30 \mathrm{~s}$ & $30 \mathrm{~s}$ & & \\
\hline $\begin{array}{c}\text { Rest } \\
\text { (Darkness) }\end{array}$ & Stimulus & Rest & Stimulus & Rest & Stimulus & Rest & Stimulus & Rest & Stimulus & Rest & Stimulus \\
\hline $30 \mathrm{~s}$ & $30 \mathrm{~s}$ & $30 \mathrm{~s}$ & $30 \mathrm{~s}$ & $30 \mathrm{~s}$ & $30 \mathrm{~s}$ & $30 \mathrm{~s}$ & $30 \mathrm{~s}$ & $30 \mathrm{~s}$ & $30 \mathrm{~s}$ & $30 \mathrm{~s}$ & $30 \mathrm{~s}$ \\
\hline
\end{tabular}

Fig. 3. Experimental procedure for Experiment 1 (A) and 2 (B). (A) Randomized after $30 \mathrm{~s}$ in the dark state before the experimental illumination (including dark field) is provided. Experimental lighting conditions: dark field, 2,600 K-30 Ix, 3,400 K-30 Ix, 4,700 K-30 Ix, 5,500 K-30 Ix, 6,000 K-30 Ix, 2,600 K-400 Ix, 3,200 K-400 Ix, 4,400 K-400 Ix, 5,400 K$400 \mathrm{Ix}, 6,000 \mathrm{~K}-400 \mathrm{Ix}$. (B) Experimental lighting and visual stimulation were performed at random. Lighting: 2,600 K$30 \mathrm{~lx}, 4,400 \mathrm{~K}-400 \mathrm{~lx}, 6,000 \mathrm{~K}-400 \mathrm{~lx}$.

Table 2. Lighting conditions for Experiment 2

\begin{tabular}{|c|c|c|c|c|c|c|c|}
\hline \multirow{3}{*}{ No. } & \multirow{3}{*}{ Lighting label } & \multicolumn{2}{|c|}{$\mathrm{CIE}^{\mathrm{z}}$} & \multirow{3}{*}{$\mathrm{Ra}^{\mathrm{y}}$} & \multirow{3}{*}{$\begin{array}{c}\text { Dominant } \\
\text { wavelength }^{\mathrm{x}} \\
(\mathrm{nm})\end{array}$} & \multirow{3}{*}{$\begin{array}{c}\text { Peak } \\
\text { wavelength }^{\mathrm{w}} \\
(\mathrm{nm})\end{array}$} & \multirow{3}{*}{$\begin{array}{c}\text { Excitation } \\
\text { purity }^{\mathrm{v}} \\
(\%)\end{array}$} \\
\hline & & \multicolumn{2}{|c|}{ Chromaticity coordinates values } & & & & \\
\hline & & $\mathrm{x}$ & $\mathrm{y}$ & & & & \\
\hline 1 & $2600 \mathrm{~K}-30 \mathrm{~lx}$ & 0.466 & 0.414 & 85 & 584 & 616 & 0.641 \\
\hline 2 & $4400 \mathrm{~K}-400 \mathrm{~lx}$ & 0.361 & 0.358 & 88 & 579 & 448 & 0.160 \\
\hline 3 & $6000 \mathrm{~K}-400 \mathrm{~lx}$ & 0.323 & 0.336 & 85 & 495 & 449 & 0.325 \\
\hline
\end{tabular}

${ }^{\mathrm{z}} \mathrm{CIE}$, Commission Internationale de l'Eclairage.

${ }^{\mathrm{y}} \mathrm{Ra}$ : Color rendering index (CRI): CRI is the degree of color seen by the naked eye from two light sources: light source and sunlight. xDominant wavelength: Method of characterizing any light mixture in terms of monochromatic spectral light that evokes an identical perception of hue.

weak wavelength: The wavelength at which the spectrum reaches its highest intensity.

${ }^{v}$ Excitation purity: The distance from the illuminant point to the reference point, divided by that from the illuminant point to the spectrum locus (relative distance of an object's chromaticity coordinate from the white point to the exterior of the chromaticity diagram). 


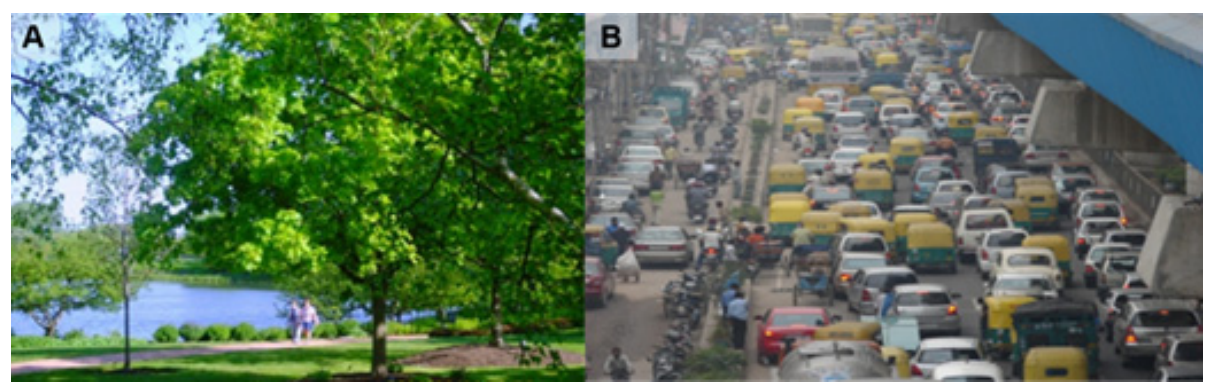

Fig. 4. Visual stimuli with positive (A, green landscape) and negative (B, traffic congestion) images used in Experiment 2.

Table 3. Electroencephalographic (EEG) responses in darkness and under 10 LED color temperature lighting conditions (relative power [\%]) in Experiment 1

\begin{tabular}{|c|c|c|c|c|c|c|c|c|c|c|c|c|c|}
\hline Lobe & Channel & Wave $^{\mathrm{z}}$ & $\begin{array}{c}\text { Darkness } \\
(0 \mathrm{~K}-0 \text { lx })\end{array}$ & $\begin{array}{c}2600 \mathrm{~K}- \\
30 \mathrm{~lx}\end{array}$ & $\begin{array}{c}3400 \mathrm{~K}- \\
30 \mathrm{~lx}\end{array}$ & $\begin{array}{c}4700 \mathrm{~K}- \\
30 \mathrm{~lx}\end{array}$ & $\begin{array}{c}5500 \mathrm{~K}- \\
30 \mathrm{~lx}\end{array}$ & $\begin{array}{c}6000 \mathrm{~K}- \\
30 \mathrm{~lx}\end{array}$ & $\begin{array}{c}2600 \mathrm{~K}- \\
400 \mathrm{~lx}\end{array}$ & $\begin{array}{c}3200 \mathrm{~K}- \\
400 \mathrm{~lx}\end{array}$ & $\begin{array}{c}4400 \mathrm{~K}- \\
400 \mathrm{~lx}\end{array}$ & $\begin{array}{c}5400 \mathrm{~K}- \\
400 \mathrm{~lx}\end{array}$ & $\begin{array}{r}6000 \mathrm{~K}- \\
400 \mathrm{~lx}\end{array}$ \\
\hline \multirow{2}{*}{$\begin{array}{c}\text { Central } \\
\text { lobe }\end{array}$} & \multirow{2}{*}{$\mathrm{Cz}$} & $\alpha$ & $37.2 b^{y}$ & $29.4 \mathrm{ab}$ & $29.9 \mathrm{ab}$ & $28.2 \mathrm{ab}$ & $30.8 \mathrm{ab}$ & $28.5 \mathrm{ab}$ & $25.1 \mathrm{a}$ & $24.4 \mathrm{a}$ & $25.6 \mathrm{a}$ & $22.8 \mathrm{a}$ & $24.5 \mathrm{a}$ \\
\hline & & $\alpha 2$ & $24.7 \mathrm{c}$ & $19.8 \mathrm{abc}$ & $20.1 b c$ & $19.8 \mathrm{abc}$ & $21.8 \mathrm{bc}$ & $19.7 \mathrm{abc}$ & 16.9abc & 16.1ab & $16.6 \mathrm{ab}$ & $14.4 \mathrm{ab}$ & $11.9 \mathrm{a}$ \\
\hline \multirow{2}{*}{$\begin{array}{c}\text { Frontal } \\
\text { lobe }\end{array}$} & $\mathrm{F} 4$ & $\alpha$ & $32.7 \mathrm{c}$ & 26.9abc & $26.1 \mathrm{abc}$ & $26.6 a b c$ & $28.6 \mathrm{bc}$ & $26.9 \mathrm{abc}$ & $23.4 \mathrm{ab}$ & $22.4 \mathrm{ab}$ & $22.3 \mathrm{ab}$ & $20.5 \mathrm{ab}$ & $19.1 \mathrm{a}$ \\
\hline & $\mathrm{Fz}$ & $\alpha$ & $37.8 \mathrm{~b}$ & $29.5 \mathrm{ab}$ & $28.2 \mathrm{a}$ & $28.2 \mathrm{a}$ & $30.1 \mathrm{ab}$ & $29.3 \mathrm{ab}$ & $25.9 \mathrm{a}$ & $24.7 \mathrm{a}$ & $26.7 \mathrm{a}$ & $23.3 \mathrm{a}$ & $23.2 \mathrm{a}$ \\
\hline \multirow{6}{*}{$\begin{array}{c}\text { Occipital } \\
\text { lobe }\end{array}$} & \multirow{2}{*}{$\mathrm{O} 1$} & $\alpha$ & $45.5 b$ & $40.1 \mathrm{ab}$ & $39.1 \mathrm{ab}$ & $38.8 \mathrm{ab}$ & $40.7 \mathrm{ab}$ & $38.6 \mathrm{ab}$ & $33.3 \mathrm{a}$ & $32.6 \mathrm{a}$ & $33.1 \mathrm{a}$ & $30.4 \mathrm{a}$ & $30.0 \mathrm{a}$ \\
\hline & & $\alpha 2$ & $34.3 \mathrm{c}$ & $29.2 \mathrm{abc}$ & 28.9abc & $27.6 \mathrm{abc}$ & $30.1 b c$ & $26.7 \mathrm{abc}$ & $22.1 \mathrm{ab}$ & $22.2 \mathrm{ab}$ & $22.5 \mathrm{ab}$ & $20.3 \mathrm{ab}$ & $19.2 \mathrm{a}$ \\
\hline & \multirow{2}{*}{$\mathrm{O} 2$} & $\alpha$ & $43.9 \mathrm{c}$ & $38.8 \mathrm{abc}$ & $38.7 \mathrm{abc}$ & $38.2 \mathrm{abc}$ & $40.8 b c$ & $38.1 \mathrm{abc}$ & $32.8 \mathrm{abc}$ & $31.3 \mathrm{ab}$ & 32.9abc & $30.0 \mathrm{ab}$ & $28.1 \mathrm{a}$ \\
\hline & & $\alpha 2$ & $33.3 \mathrm{c}$ & $28.7 \mathrm{abc}$ & 28.9abc & $27.8 \mathrm{abc}$ & $30.6 \mathrm{bc}$ & $26.8 \mathrm{abc}$ & $22.4 \mathrm{ab}$ & $22.7 \mathrm{ab}$ & 23.0abc & $20.6 \mathrm{ab}$ & $18.9 \mathrm{a}$ \\
\hline & \multirow{2}{*}{$\mathrm{Oz}$} & $\alpha$ & $45.2 \mathrm{c}$ & $39.6 \mathrm{abc}$ & $39.0 \mathrm{abc}$ & $38.7 \mathrm{abc}$ & $41.9 \mathrm{bc}$ & $39.0 \mathrm{abc}$ & $33.1 \mathrm{ab}$ & $32.1 \mathrm{ab}$ & $33.1 \mathrm{ab}$ & $31.2 \mathrm{ab}$ & $29.4 \mathrm{a}$ \\
\hline & & $\alpha 2$ & $33.2 \mathrm{c}$ & $29.3 b c$ & $29.1 \mathrm{bc}$ & $28.0 \mathrm{abc}$ & $30.8 \mathrm{bc}$ & $27.2 \mathrm{abc}$ & $22.3 \mathrm{ab}$ & $22.4 \mathrm{ab}$ & $22.7 \mathrm{ab}$ & $21.6 \mathrm{ab}$ & $18.2 \mathrm{a}$ \\
\hline \multirow{3}{*}{$\begin{array}{c}\text { Temporal } \\
\text { lobe }\end{array}$} & \multirow{3}{*}{ T6 } & $\alpha$ & $44.1 \mathrm{~b}$ & $39.0 \mathrm{ab}$ & $37.5 \mathrm{ab}$ & $38.5 \mathrm{ab}$ & $41.0 \mathrm{~b}$ & $38.0 \mathrm{ab}$ & $33.6 \mathrm{ab}$ & $33.3 \mathrm{ab}$ & $33.5 \mathrm{ab}$ & $28.6 \mathrm{a}$ & $27.9 \mathrm{a}$ \\
\hline & & $\alpha 2$ & $33.5 \mathrm{c}$ & $26.5 \mathrm{abc}$ & $26.1 \mathrm{abc}$ & $26.5 \mathrm{abc}$ & $29.7 b c$ & $25.0 \mathrm{abc}$ & $22.3 \mathrm{ab}$ & $21.5 \mathrm{ab}$ & $22.2 \mathrm{ab}$ & $17.3 \mathrm{a}$ & $21.4 \mathrm{ab}$ \\
\hline & & $\beta 2$ & $2.65 \mathrm{a}$ & $3.22 \mathrm{a}-\mathrm{d}$ & $3.19 \mathrm{abc}$ & $3.15 \mathrm{abc}$ & $2.71 \mathrm{ab}$ & 3.53a-d & $3.60 \mathrm{~cd}$ & $3.58 \mathrm{bcd}$ & 3.36a-d & $3.49 \mathrm{a}-\mathrm{d}$ & $4.08 \mathrm{~d}$ \\
\hline
\end{tabular}

${ }^{\mathrm{z}} \alpha(8-12 \mathrm{~Hz}), \alpha 2(10-12 \mathrm{~Hz}), \beta 2(25-30 \mathrm{~Hz})$

${ }^{\mathrm{y}}$ Means within rows followed by different letters are significantly different as per Duncan's multiple range test at $\mathrm{p} \leq 0.05$.

\section{Research Limitations}

This study investigated EEG and HRV responses under random lighting conditions in 24 university students who voluntarily participated in the experiment. The number of subjects participating in the experiment was very small, which makes it difficult to generalize the results of this study.

Another limitation of this study is that the subjects were not completely free of the effects of the previous illumination, as each subject was subjected to multiple lighting conditions (even though intermediate breaks in illumination were used) wthin a limited time.

\section{Results}

\section{Experiment 1}

In Experiment 1, there were significant differences between the darkness and high illumination level (400 lx) conditions at $7(\mathrm{Cz}, \mathrm{F} 4, \mathrm{Fz}, \mathrm{O} 1, \mathrm{O} 2, \mathrm{Oz}$, and T6) of 30 channels. There were also significant differences in the alpha wave $(8-12 \mathrm{~Hz})$ and beta wave $(12-25 \mathrm{~Hz})$ activities at channel T6. Specifically, alpha wave and alpha wave $2(10-12 \mathrm{~Hz})$ activities were significantly lower under the high illumination (400 lx) conditions than under dark conditions. The beta wave activity decreased as the color temperature increased, decreased in the darkness and low illumination (30 lx) conditions, and increased in the 2,600 and 6,000 K low illumination (30 lx) conditions (Table 3).

\section{Experiment 2}

\section{Analysis by channel (brain region)}

Positive (green landscape) and negative (traffic congestion) stimuli were presented under three LED color temperature conditions $(30 \mathrm{~lx}-2,600 \mathrm{~K}, 4001 \mathrm{x}-4,400 \mathrm{~K}$, and $4001 \mathrm{x}-6,000 \mathrm{~K})$. In the $30 \mathrm{~lx}-2,600 \mathrm{~K}$ condition, negative stimulus produced a higher theta wave $(4-8 \mathrm{~Hz})$ activity in 
Table 4. Electroencephalographic (EEG) responses to positive and negative images under the 2,600 K-30 Ix, 4,400 K$400 \mathrm{~lx}$, and 6,000 K-400 Ix lighting conditions in Experiment 2

\begin{tabular}{|c|c|c|c|c|c|c|}
\hline \multirow[t]{2}{*}{ Lighting condition } & \multirow[t]{2}{*}{ Channel } & \multirow[t]{2}{*}{ Wave $^{\mathrm{z}}$} & \multicolumn{2}{|c|}{$\begin{array}{l}\text { EEG response to images } \\
\text { (relative power [\%]) }\end{array}$} & \multirow[t]{2}{*}{$\mathrm{t}$} & \multirow[t]{2}{*}{$p$} \\
\hline & & & Positive & Negative & & \\
\hline \multirow[t]{6}{*}{$2600 \mathrm{~K}-30 \mathrm{~lx}$} & $\mathrm{P} 4$ & $\theta$ & $15.16(6.86)$ & $16.68(7.36)$ & -2.797 & $.011^{*}$ \\
\hline & $\mathrm{O} 2$ & $\theta$ & $17.43(5.98)$ & $19.33(5.89)$ & -2.528 & $.020^{*}$ \\
\hline & $\mathrm{OZ}$ & $\theta$ & $17.36(5.92)$ & $19.05(5.93)$ & -2.507 & $.020^{*}$ \\
\hline & F3 & $\theta$ & $18.53(8.52)$ & $20.00(9.17)$ & -2.448 & $.023^{*}$ \\
\hline & $\mathrm{Ft} 7$ & $\theta$ & $15.35(6.17)$ & $16.61(6.44)$ & -2.133 & $.045^{*}$ \\
\hline & $\mathrm{T} 6$ & $\theta$ & $16.65(5.69)$ & $18.10(5.73)$ & -2.282 & $.033^{*}$ \\
\hline \multirow[t]{2}{*}{4400 K-400 lx } & $\mathrm{Fc} 4$ & $\beta 2$ & $4.58(1.50)$ & $5.03(1.81)$ & -2.123 & $.046^{*}$ \\
\hline & $\mathrm{Pz}$ & $\beta 2$ & $3.32(1.15)$ & $3.62(1.09)$ & -2.381 & $.027^{*}$ \\
\hline \multirow[t]{9}{*}{6000 K-400 lx } & $\mathrm{C} 4$ & $\alpha 2$ & $14.56(9.51)$ & $12.87(8.36)$ & 2.616 & $.016^{*}$ \\
\hline & $\mathrm{Cp} 4$ & High $\beta$ & $3.34(2.35)$ & $3.96(2.56)$ & -2.217 & $.038^{*}$ \\
\hline & & $\alpha 2$ & $16.77(14.05)$ & $14.89(12.16)$ & 2.414 & $.025^{*}$ \\
\hline & Fp1 & $\gamma$ & $5.49(4.46)$ & $6.34(5.20)$ & -2.517 & $.020^{*}$ \\
\hline & & $\gamma 1$ & $3.29(2.65)$ & $3.88(3.26)$ & -2.610 & $.160^{\mathrm{NS}}$ \\
\hline & & $\gamma_{2}$ & $2.19(1.83)$ & $2.46(1.95)$ & -2.104 & $.048^{*}$ \\
\hline & P4 & $\beta 1$ & $8.91(3.71)$ & $7.89(2.69)$ & 2.230 & $.037^{*}$ \\
\hline & $\mathrm{T} 3$ & $\beta 1$ & $7.01(2.00)$ & $6.40(1.71)$ & 2.210 & $.038^{*}$ \\
\hline & Tp8 & $\operatorname{High} \beta$ & $3.76(2.29)$ & $4.26(2.28)$ & -2.101 & $.048^{*}$ \\
\hline
\end{tabular}

${ }^{7} \theta(4-8 \mathrm{~Hz}), \alpha 2(10-12 \mathrm{~Hz}), \beta 1(12-15 \mathrm{~Hz})$, high $\beta(15-18 \mathrm{~Hz}), \beta 2(25-30 \mathrm{~Hz}), \gamma(30-40 \mathrm{~Hz}), \gamma 1(30-35 \mathrm{~Hz}), \gamma 2(35-40 \mathrm{~Hz})$.

NS, ${ }^{*}$ Nonsignificant or significant at $\mathrm{p}<0.05$, one-way analysis of variance.

the parietal, occipital, frontal, and temporal lobes than positive stimulus (Table 4). The theta wave activity is the main brainwave in states of mental tranquility, such as during meditation, but is associated with dormancy and decreased brain function in the awake state. For example, students who are inattentive and have low academic grades often exhibit strong theta waves. Conversely, theta wave control is associated with improved attention and concentration.

In the $400 \mathrm{~lx}-4,400 \mathrm{~K}$ lighting condition, negative stimulus produced a higher beta $2\left(15^{-18} \mathrm{~Hz}\right)$ activity in the frontal and parietal lobes than positive stimulus (Table 4). Beta waves (12-25 Hz) are associated with an increased level of awareness and can be an indication for sedative use to treat anxiety, migraine, and insomnia.

In the $400 \mathrm{~lx}-6,000 \mathrm{~K}$ lighting condition, positive stimulus produced high alpha $2(10-12 \mathrm{~Hz})$ activity, and negative stimulus produced high beta $1(12-15 \mathrm{~Hz})$, beta 2 (25-30 Hz), gamma (30-40 Hz), gamma $1(30-35 \mathrm{~Hz})$, and gamma $2(35-40 \mathrm{~Hz})$ activities in the central, frontal, parie- tal, and temporal lobes (Table 4).

\section{HRV results}

The SDNN (a stress coping ability index) and RMSSD (an HR stability index) were high when positive stimulus was presented in the dark. The SDNN also increased after positive stimulus was presented in the $400 \mathrm{~lx}-4,400 \mathrm{~K}$ condition (Table 5).

\section{Discussion}

This study examined EEG and HRV responses to various LED color temperature conditions in 24 university students; in Experiment 1, the alpha wave (8-12 Hz) activity was high under low illumination $(30 \mathrm{~lx})$ conditions, whereas the beta wave (12-25 Hz) activity was high under high illumination (400 lx) conditions, regardless of color temperature. In Experiment 2, the alpha wave $\left(8^{-12} \mathrm{~Hz}\right)$ 
Table 5. Heart rate variability (HRV) because of green landscape (positive) and traffic congestion (negative) images in the darkness and under three light-emitting diode color temperature lighting conditions in Experiment 2

\begin{tabular}{|c|c|c|c|c|}
\hline \multirow{2}{*}{ Item $^{\mathrm{z}}$} & \multirow{2}{*}{ Image } & \multicolumn{3}{|c|}{ HRV (relative power [\%]) } \\
\hline & & $2600 \mathrm{~K}-30 \mathrm{~lx}$ & $4400 \mathrm{~K}-400 \mathrm{~lx}$ & $6000 \mathrm{~K}-400 \mathrm{~lx}$ \\
\hline \multirow{4}{*}{ SDNN } & Positive & $85.38(55.88)$ & $77.73(33.64)$ & $82.29(58.39)$ \\
\hline & Negative & $74.38(50.84)$ & $60.50(29.36)$ & $75.82(48.14)$ \\
\hline & $\mathrm{t}$ & 1.052 & 3.363 & 0.649 \\
\hline & $\mathrm{P}$ & $0.305^{\mathrm{NS}}$ & $0.003^{* *}$ & $0.524^{\mathrm{NS}}$ \\
\hline \multirow{4}{*}{ HR } & Positive & $71.00(7.82)$ & $69.50(8.77)$ & $71.43(8.56)$ \\
\hline & Negative & $71.20(7.33)$ & $70.82(7.08)$ & $69.83(7.34)$ \\
\hline & $\mathrm{t}$ & -0.293 & -0.899 & 2.062 \\
\hline & $\mathrm{P}$ & $0.772^{\mathrm{NS}}$ & $0.379^{\mathrm{NS}}$ & $0.052^{\mathrm{NS}}$ \\
\hline \multirow{4}{*}{ RMSSD } & Positive & $78.63(70.02)$ & $66.09(43.68)$ & $74.57(71.70)$ \\
\hline & Negative & $66.85(57.42)$ & $73.12(90.13)$ & $66.42(50.19)$ \\
\hline & $\mathrm{t}$ & 0.729 & -0.337 & 0.505 \\
\hline & $\mathrm{P}$ & $0.474^{\mathrm{NS}}$ & $0.739^{\mathrm{NS}}$ & $0.619^{\mathrm{NS}}$ \\
\hline \multirow{4}{*}{$\mathrm{LF} / \mathrm{HF}$} & Positive & $1.63(1.69)$ & $2.90(2.91)$ & $1.83(1.58)$ \\
\hline & Negative & $2.01(2.47)$ & $1.94(2.44)$ & $2.10(1.98)$ \\
\hline & $\mathrm{t}$ & -0.668 & 1.463 & -0.671 \\
\hline & $P$ & $0.511^{\mathrm{NS}}$ & $0.158^{\mathrm{NS}}$ & $0.509^{\mathrm{NS}}$ \\
\hline
\end{tabular}

${ }^{\mathrm{z}} \mathrm{HR}$, heart rate; LF/HF, low-frequency to high-frequency ratio; RMSSD, root mean square of the standard deviation; SDDN, standard deviation of the $\mathrm{N}-\mathrm{N}$ interval.

${ }_{\mathrm{NS},{ }^{* *}}$ Nonsignificant or significant at $p<.01$, via one-way analysis of variance.

activity was high in response to positive image stimulus under dark conditions, while the beta $(25-30 \mathrm{~Hz})$ and gamma wave $(30-40 \mathrm{~Hz})$ activities were high in response to negative image stimulus under $400 \mathrm{~lx}$ illumination. The theta wave (4-8 Hz) activity was high in response to negative image stimulus in the $30 \mathrm{~lx}-2,600 \mathrm{~K}$ condition. With regard to HRV, the SDNN and RMSSD were high in response to positive image stimulus under dark conditions. This result suggests that positive image stimulus under lighting conditions close to darkness may be effective at reducing stress. In general, the responses to positive visual stimuli in the dark, low color temperatures, and low illumination levels were positive, whereas the responses to negative visual stimuli under high color temperatures and illumination levels were negative.

Experiment 1, which investigated EEG under dark and 10 lighting conditions, showed that the EEG frequency band alpha wave was significantly higher in the darkness. The lower the illuminance (brightness) at the same color temperature conditions, the higher the alpha wave activity.
There were no significant differences in brain activity, including alpha waves, accompanying changes in color temperature $(2,600,3,200 / 3,400,4,400 / 4,700,5,400 / 5,500$, and $6,000 \mathrm{~K}$ ) under the same level of illumination. Therefore, several meaningful lighting conditions in Experiment 1 were selected to investigate changes in the EEG and heart rate variability according to positive/negative image stimulation.

In Experiment 2, negative images at $301 \mathrm{x}-2,600 \mathrm{~K}$ showed higher theta waves (creativity, deep connection with meditation) than positive images. In the range of 400 $1 \mathrm{x}-4,400 \mathrm{~K}$, the beta 2 (stress, tension) activity was high upon negative image stimulation. In the range of $400 \mathrm{~lx}^{-}$ $6,000 \mathrm{~K}$, gamma wave, beta, and alpha 2 , and beta 1 (concentration) activities were high upon negative image stimulation. In the HRV results, SDNN, an indicator of stress coping ability, was found to be high for positive image stimulation, and there were no significant differences because of lighting conditions.

In conclusion, as per the EEG and HRV, higher color 
temperatures $(4,400-6,000 \mathrm{~K})$ under the same illuminance (400 lx) led to more positive effects of positive images and more negative effects of negative images.

Under low light and low color temperatures (30 lx $-2,600$ $\mathrm{K})$, theta wave activity was higher than beta and gamma wave activities upon negative image stimulation, suggesting that lighting control under stressful situations can emotionally relieve tension.

Our evaluation of the biosignal responses to color temperatures and illumination levels revealed that the illumination level had a significant effect on alpha wave activity. The changes in response to lighting conditions were dependent on the stimulus conditions in Experiment 2. In a study by Ji et al. (2008), the stability among sensibility factors was high when the color temperatures were low, which is consistent with our findings in Experiment 2. Accordingly, this study demonstrates that low illumination levels may alleviate tension, and darkness is associated with a high level of relaxation. The presentation of positive visual stimuli in darkness reduced physiological indicators of stress, suggesting that these lighting contexts may be useful for stress management. In contrast, negative visual stimuli under high illumination and color temperature conditions appeared to promote stress or displeasure, indicating that prolonged exposure to high illumination levels and color temperatures may lead to higher levels of stress.

In addition, the positive image used in Experiment 2, showing the green color of trees and forests, is thought to have influenced the results. Looking to the literature to clarify color-emotion associations, Kaya and Epps (2004) evaluated the association of colors with positive emotions, including feelings of relaxation, followed by happiness, comfort, peace, and hope (Kaya and Epps, 2004). Green was associated with nature and trees, thus producing feelings of comfort and soothing emotions (Kaya and Epps, 2004). Minguillon et al. (2017) reported that blue lighting accelerated the relaxation process after stress in comparison with conventional white lighting. Our observations support the relationship between the color of light and stress and the observations reported in previous works (Minguillon et al., 2017; Lee et al., 2018).

\section{Conclusion}

Lighting is an important part of our daily life; thus, properly controlling the lighting environment - that is, illuminance and color temperature - can have a substantial effect on improving mental health and work efficiency in everyday life. We examined electroencephalography (EEG) and heart rate variability (HRV) responses to various lighting conditions in 25 university students. EEG was performed in darkness and when the room was illuminated with 10 different light-emitting diode (LED) color temperatures, while the EEG and HRV responses to green landscape / traffic congestion image stimuli were measured in darkness and during room illumination with three different LED color temperatures.

We found a significant difference between darkness and high LED illumination (400 lx) at 7 (CZ, F4, FZ, O1, $\mathrm{O} 2, \mathrm{OZ}$, and $\mathrm{T} 6$ ) of 30 channels, while the alpha wave activity was increased during darkness. In the second experiment, the green landscape image stimuli in the $30 \mathrm{~lx}^{-}$ $2600 \mathrm{~K}$ lighting condition elicited theta wave activity on the EEG, whereas traffic congestion image stimuli under high LED illumination elicited high beta and gamma wave activities. Moreover, the subjects exhibited better stress coping ability and heart rate stability in response to green landscape image stimuli under illuminated conditions, according to their HRV.

These results suggest that lower color temperatures and illumination levels alleviate tension, and that viewing green landscape image stimuli at low illumination, or in darkness, is effective at reducing stress. Conversely, high illumination levels and color temperatures are likely to increase tension and stress in response to traffic congestion image stimuli. Particularly in situations when going out is not possible, such as during the COVID-19 pandemic, our findings suggest that it could be beneficial for mental health to view green plant images on a TV, PC, smartphone, etc. under appropriate lighting at home. In the future, it will be possible to improve quality of life by examining the effect of more colorful garden or landscape photos on brain waves and using photos suitable for each situation or space. 


\section{References}

Bulgari, R., A. Petrini, G. Cocetta, C. Nicoletto, A. Ertani, P. Sambo, A. Ferrante, and S. Nicola. 2021. The impact of COVID-19 on horticulture: Critical issues and opportunities derived from an unexpected occurrence. Horticulturae 7(6):124. https://doi.org/10.3390/horticulturae7060124

Chae, J. and M. Cho. 2021. Changes in the cultural trend of use by type of green infrastructure before and after COVID-19 using blog text mining in Seoul. J. People Plants Environ. 24:415-427. https://doi.org/10.11628/ksppe. 2021.24.4.415

Chebat, J.C. and R. Michon. 2003. Impact of ambient odors on mall shoppers' emotions, cognition, and spending: A test of competitive causal theories. J. Business Res. 56:529-539. https://doi.org/10.1016/S0148-2963(01)00247-8

Ergenoglu, T., D. Tamer, B. Zubeyir, E. Mehmet, B. Huseyin, and U. Yagiz. 2004. Alpha rhythm of EEG modulates visual detection performance in humans. Cogn. Brain Res. 20(3):376-383. https://doi.org/10.1016/j.cogbrainres. 2004.03.009

Higuchi, S., Y. Lin, J. Qiu, Y. Zhang, M. Ohashi, S.I. Lee, S. Kitamura, and A. Yasukouchi. 2021. Is the use of high correlated color temperature light at night related to delay of sleep timing in university students? A crosscountry study in Japan and China. J. Physiol. Anthropol. 40:1-8. https://doi.org/10.1186/s40101-021-00257-x

Ji, S.D., S.H. Lee, G.J. Choi, J.G. Park, and C.H. Kim. 2008. Sensibility evaluation on the correlated color temperature in white LED lighting. J. Korean Inst. Illuminating Electr. Install. Eng. 22(4):1-12. https://doi.org/10.5207/ JIEIE.2008.22.4.001

Jung, H.J., T.S. Park, B.H. Lee, S.H. Yun, W.Y. Lee, and W.B. Kim. 2010. Recent trend in measurement techniques of emotion science. Korean J. Sci. Emot. Sensibil. 13(1):235-242.

Kaiser, P.K. 1984. Physiological response to color: A critical review. Color Res. Appl. 9(1):29-36. https://doi.org/10.1002/col.5080090106

Kaya, N. and H.H. Epps. 2004. Color-emotion associations: Past experience and personal preference. In J.L. Caivano (Ed.), Proceedings of the AIC 2004 Color and Paints, Interim Meeting of the International Colour Association (pp. 31-34). Porto Alegre, Brazil, 3-5 November 2004.
Korea Evaluation Institute of Industrial Technology (KEIT). 2013. LED-Photonics. In KEIT PD Issue Report, Special 18 (pp. 70-75). Daegu, Korea: KEIT.

Korean National Committee of International Commission on Illumination (KCIE). 2009. Introduction to Lighting Design (pp. 12-13). Seoul, Korea; Kidari Publishing Co.

Lang, P.J. and M. Bradley. 2010. Emotion and the motivational brain. Biol. Psychol. 84(3):437-450. https://doi.org/10.1016/j.biopsycho.2009.10.007

Larcher, F., E. Pomatto, L. Battisti, P. Gullino, and M. Devecchi. 2021. Perceptions of urban green areas during the social distancing period for COVID-19 containment in Italy. Horticulturae 7(3):55. https://doi.org/10.3390/ horticulturae 7030055

Lee, B. and P. Yeon. 2021. An EDA analysis of Seoul metropolitan area's mountain usage patterns of users in their 20 30s after COVID-19 occurrence. J. People Plants Environ. 24:229-244. https://doi.org/10.11628/ksppe. 2021.24.2.22

Lee, M.J., W. Oh, J. Kim, and J.S. Jang. 2018. Colors of the LED on stress response of workers after a flower arrangement activity. Hortic. Sci. Technol. 36:435-443. https://doi.org/10.12972/kjhst.20180043

Manav, B. 2007. An experimental study on the appraisal of the visual environment at offices in relation to colour temperature and illuminance. Build. Environ. 42:979983. https://doi.org/10.1016/j.buildenv.2005.10.022

Martin, G.N. 1998. Human electroencephalographic EEG. Response to olfactory stimulation two experiments using the aroma of food. Int. J. Psychophysiol. 30(3):287-302. https://doi.org/10.1016/S0167-8760(98)00025-7

Minguillon, J., M.A. Lopez-Gordo, D.A. Renedo-Criado, M.J. Sanchez-Carrion, and F. Pelayo. 2017. Blue lighting accelerates post-stress relaxation: Results of a preliminary study. PLoS ONE 12:e0186399. https://doi.org/10.1371/journal.pone.0186399

Mulholland, T. 1973. Objective EEG methods for studying covert shifts of visual attention. In F.J. McGuigan and R.A. Schoonauer (Eds.), The Psychophysiology of Thinking (pp. 109-151). New York, USA: Academic Press.

Park, J.Y., R.Y. Ha, V. Ryu, E. Kim, and Y.C. Jung. 2013. Effects of color temperature and brightness on electroencephalogram alpha activity in a polychromatic light- 
emitting diode. Clin. Psychopharmacol. Neurosci. 11(3): 126. https://doi.org/10.9758/cpn.2013.11.3.126

Parsons, R. 2012. Environmental psychophysiology. In J.T. Cacioppo, L.G. Tassinary, and G. Berntson (Eds.), Handbook of Psychophysiology (pp. 752-786). Cambridge, UK.: Cambridge University Press. https://doi.org/10. 1017/CBO9780511546396.032

Schneider, S., V. Brümmer, T. Abel, C.D. Askew, and H.K. Strüder. 2009. Changes in brain cortical activity measured by EEG are related to individual exercise preferences. Physiol. Behav. 98(4):447-452. https://doi.org/10.1016/j.physbeh.2009.07.010

Stone, N.J. 2001. Designing effective study environments. J. Environ. Psychol. 21:179-190. https://doi.org/10.1006/ jevp.2000.0193
Suk, H.J. and H. Irtel. 2010. Emotional response to color across media. Color Res. Appl. 35:64-77. https://doi.org/ $10.1002 / \mathrm{col} .20554$

Suk, H.J., E.S. Lee, G.M. Kim, and S.H. Han. 2012. The affective effect of odor products in relaxation and working contexts. Sci. Emot. Sensit. 14(3):395-402.

Sunwoo, J.S., S. Lee, K.S. Cha, T.J. Kim, J.I. Byun, and K.Y. Jung. 2017. Differential effect of light emitting diode light on electroencephalographic oscillations in young and old healthy subjects. J. Sleep Med. 14(2):61-69. https://doi.org/10.13078/jsm.17009

Zion-Golumbic, E., T. Golan, D. Anaki, and S. Bentin. 2008. Human face preference in gamma-frequency EEG activity. NeuroImage 39:1980-1987. https://doi.org/10.1016/ j.neuroimage. 2007.10 .025 